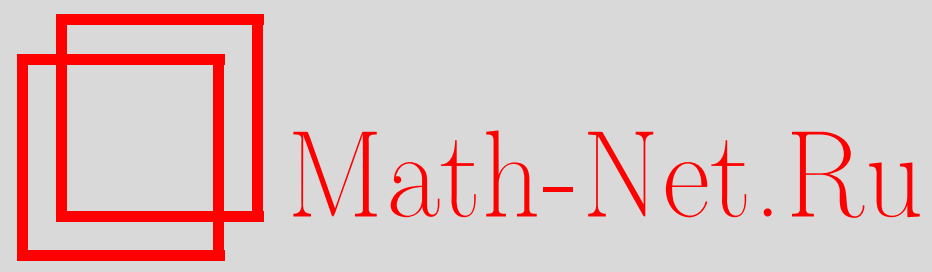

А. В. Пенской, Канонически сопряженные переменные для системы Вольтерра с периодическими граничными условиями, Матем. заметки, 1998, том 64, выпуск 1, 115-128

DOI: https://doi.org/10.4213/mzm1377

Использование Общероссийского математического портала Math-Net.Ru подразумевает, что вы прочитали и согласны с пользовательским соглашением http://www.mathnet.ru/rus/agreement

Параметры загрузки:

IP : 35.173 .137 .237

26 апреля 2023 г., 13:30:38

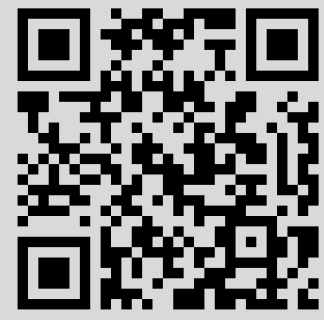


УДК 517.93

\section{КАНОНИЧЕСКИ СОПРЯЖЕННЫЕ ПЕРЕМЕННЫЕ ДЛЯ СИСТЕМЫ ВОЛЬТЕРРА \\ С ПЕРИОДИЧЕСКИМИ ГРАНИЧНЫМИ УСЛОВИЯМИ}

\section{А. В. Пенской}

В работе рассматривается система Вольтерра. Известно, что эта динамическая система является гамильтоновой по отношению к двум согласованным скобкам Пуассона (квадратичной и кубической). При помощи спектральной теории оператора Якоби для обеих скобок найдены канонически сопряженные переменные.

Библиографоия: 10 названий.

Рассмотрим систему Вольтерра, задающуюся уравнениями

$$
\dot{c}_{i}=c_{i}\left(c_{i+1}-c_{i-1}\right)
$$

где $i \in \mathbb{Z}, c_{i}(t)>0$, с периодическими граничньми условиями $c_{T+i}=c_{i}, T \in \mathbb{N}$.

Задача построения канонически сопряженных переменных при квазипериодических граничных условиях в данной статье не рассматривается и остается нерешенной.

Известно [1], [2], что данная система является гамильтоновой для двух согласованных скобок Пуассона:

1) квадратичной скобки Пуассона

$$
\left\{c_{i}, c_{j}\right\}_{1}= \begin{cases}c_{i} c_{i+1}, & \text { если } j=i+1, \\ 0 & \text { в остальных случаях }\end{cases}
$$

с гамильтонианом $H=\sum_{i=1}^{T} c_{i}$;

2) кубической скобки Пуассона

$$
\left\{c_{i}, c_{j}\right\}_{2}= \begin{cases}c_{i} c_{i+1}\left(c_{i}+c_{i+1}\right), & \text { если } j=i+1, \\ c_{i} c_{i+1} c_{i+2}, & \text { если } j=i+2, \\ 0 & \text { в остальных случаях }\end{cases}
$$

с гамильтонианом $H=\frac{1}{2} \ln \prod_{i=1}^{T} c_{i}$. 
С. В. Манаков [3] и независимо от него годом позже М. Кац и П. ван Мербеке [4] доказали интегрируемость системы Вольтерра.

Введем удобное обозначение $a_{i}=\sqrt{c_{i}}$, которое далее будет часто использоваться.

Рассмотрим оператор Якоби $l$ на пространстве последовательностей $\{y(n): n \in \mathbb{Z}\}$ :

$$
(l y)(n)=a_{n+1} y(n+1)+a_{n} y(n-1)
$$

и соответствующую спектральную задачу $(l y)(n)=\lambda y(n)$.

Канонически сопряженные переменные для обеих скобок будут построены в терминах решений этой спектральной задачи, что сближает данную работу с работой Г. Флашки и Д. Маклафлина [5], где подобным образом строятся канонически сопряженные переменные для цепочки Тоды и уравнения Кортевега-де Фриза с периодическими граничными условиями.

В работе В. Л. Верещагина [6] установлен изоморфизм между фазовыми пространствами системы Вольтерра и цепочки Тоды, который, однако, не согласован со спектральной теорией и поэтому не может быть использован для целей настоящей работы.

Рассуждения зависят от четности периода $T$; часто выкладки будут проводиться только для нечетного случая $T=2 N+1$, но отличия для четного случая $T=2 N+2$ будут оговариваться.

Рассмотрим фундаментальные решения $y_{1}(n, \lambda)$ и $y_{2}(n, \lambda)$ уравнения $l y=\lambda y$, нормированные следуюшим образом:

$$
y_{1}(-N)=1, \quad y_{1}(-N+1)=0, \quad y_{2}(-N)=0, \quad y_{2}(-N+1)=1 .
$$

В данном базисе матрица монодромии вьглядит так:

$$
U=\left(\begin{array}{cc}
y_{1}(-N+T) & y_{2}(-N+T) \\
y_{1}(-N+1+T) & y_{2}(-N+1+T)
\end{array}\right)
$$

Обозначим через $\rho$ множитель $Ф$ локе. Он удовлетворяет уравнению $\operatorname{det}(U-\rho)=0$.

Для произвольных решений $\psi_{i}$ уравнения $l \psi_{i}=\lambda_{i} \psi_{i}, i=1,2$, обозначим через $W_{k}\left(\psi_{1}, \psi_{2}\right)$ разностньй вронскиан $\psi_{1}$ и $\psi_{2}$ :

$$
W_{k}\left(\psi_{1}, \psi_{2}\right)=a_{k+1}\left(\psi_{1}(k) \psi_{2}(k+1)-\psi_{1}(k+1) \psi_{2}(k)\right) .
$$

Если $\lambda_{1}=\lambda_{2}$, то легко показать, что $W_{k}\left(\psi_{1}, \psi_{2}\right)=W_{k+1}\left(\psi_{1}, \psi_{2}\right)$, поэтому

$$
\operatorname{det} U=\frac{W_{-N+T}\left(y_{1}, y_{2}\right)}{a_{-N+1+T}}=\frac{W_{-N}\left(y_{1}, y_{2}\right)}{a_{-N+1}}=1,
$$

откуда следует, что $\rho$ удовлетворяет уравнению

$$
\rho^{2}-\Delta(\lambda) \rho+1=0
$$

где $\Delta(\lambda)=\operatorname{tr} U=y_{1}(-N+T)+y_{2}(-N+1+T)$.

Рассмотрим $\lambda$ такие, что существует ненулевое решение $y$ с нулевыми граничными условиями $y(-N, \lambda)=y(-N+T, \lambda)=0$. Все такие решения пропорциональны $y_{2}$, поэтому соответствующие $\lambda$ определяются из уравнения $y_{2}(-N+T, \lambda)=0$. 
Легко видеть, что $y_{2}(N+1, \lambda)=Q_{1}\left(\lambda^{2}\right), y_{2}(N+2, \lambda)=\lambda Q_{2}\left(\lambda^{2}\right)$, где $Q_{1}, Q_{2}-$ многочлены степени $N$, поэтому решениями в нечетном случае будут $\pm \lambda_{i}, i=1, \ldots, N$, а в четном $0, \pm \lambda_{i}, i=1, \ldots, N$. Таким образом, с точностью до знака имеем $N$ ненулевых собственных значений. Выберем знаки произвольньм образом: позднее мы увидим, что от выбора знака практически ничего не зависит.

Пусть $P(\lambda)=\Delta(\lambda) / 2, R(\lambda)=(P(\lambda)+1)(P(\lambda)-1)$. Пусть $\Gamma$ - гиперэллиптическая кривая, заданная уравнением $y^{2}=R(\lambda)$; она двулистно накрывает плоскость $\lambda$, поэтому ее точки будем обозначать парами $(\lambda, \pm)$, где \pm задает лист.

Любому значению $\lambda$ соответствуют два решения уравнения (1): $\rho^{+}$и $\rho^{-}$, причем $\rho^{+} \rho^{-}=1$. Рассмотрим решение системы уравнений

$$
\left(l \psi^{ \pm}\right)(n)=\lambda \psi^{ \pm}(n), \quad \psi^{ \pm}(n+T)=\rho^{ \pm} \psi^{ \pm}(n),
$$

нормированное условием $\psi^{ \pm}(-N)=1$. Известно [7], что две функции $\psi^{ \pm}(n, \lambda)$ на самом деле являются однозначной мероморфной функцией на гиперэллиптической кривой $\Gamma$, при этом у $\psi(n, \lambda)$ при любом $n$ вне бесконечноудаленных точек ровно $T-1$ полюсов $P_{1}, \ldots, P_{T-1}$, проекции которых на плоскость $\lambda$ - в точности $\pm \lambda_{k}$ и еше 0 в четном случае. Занумеруем их таким образом: $P_{i}=\left(\lambda_{i}, \pm\right), P_{N+i}=\left(-\lambda_{i}, \pm\right), i=1, \ldots, N$, и в четном случае $P_{2 N+1}=0$.

Введем на кривой $\Gamma$ инволюцию $\sigma$ следующим образом:

$$
\sigma(y, \lambda)= \begin{cases}(-y,-\lambda) & \text { в нечетном случае } \\ (y,-\lambda) & \text { в четном случае. }\end{cases}
$$

Известно [8], [9], что дивизор $\mathscr{D}=P_{1}+\cdots+P_{T-1}$ полюсов $\psi_{n}$, лежащих вне бесконечноудаленных точек, инвариантен относительно инволюции: $\sigma(\mathscr{D})=\mathscr{D}$.

Легко проверить, что $y_{2}\left(n, \lambda_{i}\right)$ - решение $\Phi$ локе, а $\rho_{i}=y_{2}\left(-N+1+T, \lambda_{i}\right)$ - соответствуюший множитель $\Phi$ локе. Известно [7], что конщы зон вещественны и симметричны относительно нуля, полюса $P_{i}$ лежат по одному в лакунах спектра и множители $\Phi$ локе действительны и взаимнообратны.

Пусть

$$
F_{i}=\ln \left(\left|\rho_{i}\right|\right)=\left\{\begin{array}{l}
\ln \left(\left|y_{2}\left(N+2, \lambda_{i}\right)\right|\right) \text { в нечетном случае, } \\
\ln \left(\left|y_{2}\left(N+3, \lambda_{i}\right)\right|\right) \text { в четном случае. }
\end{array}\right.
$$

Теорема 1. Координаты $\left(\lambda_{i}, 2 F_{j} / \lambda_{j}: i, j=1, \ldots, N\right)$ являются канонически сопряженными для квадратичной скобки, т.е.

$$
\left\{\lambda_{i}, \lambda_{j}\right\}_{1}=0, \quad\left\{\lambda_{i}, \frac{2 F_{j}}{\lambda_{j}}\right\}_{1}=\delta_{i, j}, \quad\left\{\frac{2 F_{i}}{\lambda_{i}}, \frac{2 F_{j}}{\lambda_{j}}\right\}_{1}=0 .
$$

ЗАмечАниЕ. Фазовое пространство имеет в нечетном случае размерность $2 N+1$, но далее будет показано, что размерность аннулятора квадратичной скобки равна 1 (соответственно $2 N+2$ и 2 в четном случае). Поэтому это полньй набор канонически сопряженных переменных.

Доказательство будет проведено для нечетного случая, так как в четном все аналогично.

Пусть $\psi_{1}, \psi_{2}$ - произвольные решения уравнения $l \psi_{i}=\lambda_{i} \psi_{i}, i=1,2$. Пусть

$$
\begin{aligned}
& W_{k}^{\uparrow}=W_{k}^{\uparrow}\left(\psi_{1}, \psi_{2}\right)=a_{k+1}\left(\lambda_{1} \psi_{1}(k) \psi_{2}(k+1)-\lambda_{2} \psi_{2}(k) \psi_{1}(k+1)\right), \\
& W_{k}^{\downarrow}=W_{k}^{\downarrow}\left(\psi_{1}, \psi_{2}\right)=a_{k+1}\left(\lambda_{2} \psi_{1}(k) \psi_{2}(k+1)-\lambda_{1} \psi_{2}(k) \psi_{1}(k+1)\right) .
\end{aligned}
$$


Лемма 1. Справедливы следующие тождества:

$$
\begin{gathered}
W_{k+1}^{\uparrow}=W_{k}^{\downarrow}, \\
W_{k}^{\downarrow}=W_{k-1}^{\uparrow}+\left(\lambda_{2}^{2}-\lambda_{1}^{2}\right) \psi_{1}(k) \psi_{2}(k), \quad W_{k+1}^{\uparrow}-W_{k-1}^{\uparrow}=\left(\lambda_{2}^{2}-\lambda_{1}^{2}\right) \psi_{1}(k) \psi_{2}(k) .
\end{gathered}
$$

ДоКАЗАТЕЛЬСТво состоит в непосредственной проверке.

Пусть $\varphi\left(n, \lambda_{i}\right)$ - удовлетворяющее нулевым граничным условиям решение, нормированное условием $\sum_{k=-N}^{N} \varphi^{2}\left(n, \lambda_{i}\right)=1 ; y\left(n, \lambda_{i}\right)$ - независимое от $y_{2}\left(n, \lambda_{i}\right)$ решение $\Phi$ локе, нормированное условием $y\left(-N, \lambda_{i}\right)=1$. Для краткости будем использовать обозначения $\varphi_{i}(n)=\varphi\left(n, \lambda_{i}\right), y_{2, i}(n)=y_{2}\left(n, \lambda_{i}\right)$ и т.д. Пусть $Z_{i}=\sum_{k=-N}^{N} y_{2, i}(k) y_{i}(k)$.

При варьировании $a_{k}$ рассмотрим вариации $\lambda_{i}$ и $F_{i}$ в предположении, что $\lambda_{i}$ остается собственным значением, для которого существует решение, удовлетворяющее нулевым граничным условиям.

Лемма 2. Вариачии $\delta \lambda_{i} u \delta F_{i}$ определяются по следующим формулам:

$$
\begin{gathered}
\delta \lambda_{i}=\sum_{k=-N}^{N} 2 \delta a_{k} \varphi_{i}(k-1) \varphi_{i}(k) \\
\delta F_{i}=\frac{1}{a_{N+2}} \sum_{k=-N}^{N} \delta a_{k}\left(2 Z_{i} \varphi_{i}(k-1) \varphi_{i}(k)-y_{2, i}(k) y_{i}(k-1)-y_{i}(k) y_{2, i}(k-1)\right) .
\end{gathered}
$$

ДоКАЗАТЕЛЬСТво. Проварьируем $l \varphi_{i}=\lambda_{i} \varphi_{i}$ :

$\delta a_{k+1} \varphi_{i}(k+1)+\delta a_{k} \varphi_{i}(k-1)+a_{k+1} \delta \varphi_{i}(k+1)+a_{k} \delta \varphi_{i}(k-1)=\delta \lambda_{i} \varphi_{i}(k)+\lambda_{i} \delta \varphi_{i}(k)$.

Умножим это равенство на $\varphi_{i}(k)$ и просуммируем по $k$ от $-N$ до $N$. Учитьвая нормировку $\varphi_{i}$ и приводя подобные члены, получим

$$
\begin{aligned}
\delta \lambda_{i}= & \sum_{k=-N}^{N}\left(\delta a_{k+1} \varphi_{i}(k+1) \varphi_{i}(k)+\delta a_{k} \varphi_{i}(k-1) \varphi_{i}(k)\right) \\
& +\sum_{k=-N}^{N}\left(-\lambda_{i} \delta \varphi_{i}(k) \varphi_{i}(k)+a_{k+1} \delta \varphi_{i}(k+1) \varphi_{i}(k)+a_{k} \delta \varphi_{i}(k-1) \varphi_{i}(k)\right) .
\end{aligned}
$$

Во втором и третьем членах второй суммы можно сделать замену индекса суммирования $k \pm 1 \mapsto k^{\prime}$, что не меняет пределов суммирования, так как $\varphi(N+1), \varphi(-N)$ и соответственно $\delta \varphi(N+1), \delta \varphi(-N)$ равны 0 . Подобные переходы типа $\sum_{k=-N}^{N} x_{k}=$ $\sum_{k=-N}^{N} x_{k+1}$ будут и далее встречаться: если они специально не оговорены, то они возможны, поскольку $x_{-N}=x_{N+1}=0$. После этого видно, что вторая сумма равна 0 , так как она равна

$$
\sum_{k=-N}^{N} \delta \varphi_{i}(k)\left(\left(l \varphi_{i}\right)(k)-\lambda_{i} \varphi_{i}(k)\right) .
$$

Проделав аналогичную замену индекса в первой сумме, получим (2). 
Проварьируем $l y_{2, i}(k)=\lambda_{i} y_{2, i}(k)$, умножим на $y_{i}(k)$ и вычтем из полученного выражения равенство $l y_{i}(k)=\lambda_{i} y_{i}(k)$, умноженное на $\delta y_{2, i}(k)$. В результате получим

$$
\begin{aligned}
& a_{k+1} \delta y_{2, i}(k+1) y_{i}(k)-a_{k+1} y_{i}(k+1) \delta y_{2, i}(k) \\
& \quad+a_{k} \delta y_{2, i}(k-1) y_{i}(k)-a_{k} y_{i}(k-1) \delta y_{2, i}(k) \\
& \quad=\delta \lambda_{i} y_{2, i}(k) y_{i}(k)-\delta a_{k+1} y_{2, i}(k+1) y_{i}(k)-\delta a_{k} y_{2, i}(k-1) y_{i}(k)
\end{aligned}
$$

Просуммируем данное выражение по $k$ от $-N+1$ до $N+1$. Слева второй и третий члены дадут 0 , так как $\delta y_{2, i}(N+1)=\delta y_{2, i}(-N)=0$, и можно произвести замену индекса суммирования подобно сделанному вьше. Слева останется

$$
\begin{aligned}
& \sum_{k=-N+1}^{N+1} a_{k+1} \delta y_{2, i}(k+1) y_{i}(k)-a_{k} y_{i}(k-1) \delta y_{2, i}(k) \\
& \quad=a_{N+2} \delta y_{2, i}(N+2) y_{i}(N+1)-a_{-N+1} y(-N) \delta y_{2, i}(-N+1) .
\end{aligned}
$$

Так как $\delta y_{2, i}(-N+1)=0$, а $y_{i}$ - решение $\Phi$ локе с множителем, обратньм к $y_{2}(N+2)$, выражение слева равно $a_{N+2} \delta y_{2, i}(N+2) / y_{2, i}(N+2)$. Видно, что слева стоит вариация $F_{i}$, умноженная на $a_{N+2}$. Теперь для завершения доказательства формулы (3) достаточно подставить в правую часть уже найденное выражение для $\delta \lambda_{i}$.

ДокАЗАТЕЛЬСТво ТЕОРЕмЫ 1. Так как в данном доказательстве упоминается только квадратичная скобка $\{\cdot, \cdot\}_{1}$, обозначим ее для простоты через $\{\cdot, \cdot\}$.

Рассмотрим $\left\{\lambda_{i}, \lambda_{j}\right\}$ :

$$
\begin{aligned}
\left\{\lambda_{i}, \lambda_{j}\right\}= & \sum_{k=-N}^{N} \frac{\delta \lambda_{i}}{\delta a_{k}}\left\{a_{k}, \lambda_{j}\right\}=\sum_{k=-N}^{N-1} \frac{\delta \lambda_{i}}{\delta a_{k}} \frac{\delta \lambda_{j}}{\delta a_{k+1}}\left\{a_{k}, a_{k+1}\right\}+\frac{\delta \lambda_{i}}{\delta a_{N}} \frac{\delta \lambda_{j}}{\delta a_{-N}}\left\{a_{N}, a_{-N}\right\} \\
& +\sum_{k=-N+1}^{N} \frac{\delta \lambda_{i}}{\delta a_{k}} \frac{\delta \lambda_{j}}{\delta a_{k-1}}\left\{a_{k}, a_{k-1}\right\}+\frac{\delta \lambda_{i}}{\delta a_{-N}} \frac{\delta \lambda_{j}}{\delta a_{N}}\left\{a_{-N}, a_{N}\right\} .
\end{aligned}
$$

После замены индекса суммирования во второй сумме $k-1 \mapsto k^{\prime}$, учитьвая равенства $\varphi(-N)=\varphi(N+1)=0$, получим

$$
\left\{\lambda_{i}, \lambda_{j}\right\}=\sum_{k=-N}^{N} a_{k} a_{k+1}\left(\varphi_{i}(k-1) \varphi_{i}(k) \varphi_{j}(k) \varphi_{j}(k+1)-\varphi_{j}(k-1) \varphi_{j}(k) \varphi_{i}(k) \varphi_{i}(k+1)\right) .
$$

Пусть $W_{k}^{\uparrow}=W_{k}^{\uparrow}\left(\varphi_{i}, \varphi_{j}\right)$. После преобразования $a_{k} \varphi(k-1)=\lambda \varphi(k)-a_{k+1} \varphi(k+1)$ и применения леммы 1 находим, что

$$
\left\{\lambda_{i}, \lambda_{j}\right\}=\sum_{k=-N}^{N} \frac{1}{\lambda_{j}^{2}-\lambda_{i}^{2}}\left(W_{k+1}^{\uparrow}-W_{k-1}^{\uparrow}\right) W_{k}^{\uparrow}
$$

что равно 0, так как $W_{N+1}^{\uparrow}=W_{-N}^{\uparrow}=0$. 
Найдем вариацию $2 F_{j} / \lambda_{j}$, пользуясь результатами леммы 2 :

$$
\begin{aligned}
\delta \frac{2 F_{j}}{\lambda_{j}}= & \frac{2}{a_{N+2} \lambda_{j}} \sum_{k=-N}^{N} \delta a_{k}\left(2 Z_{j} \varphi_{j}(k) \varphi_{j}(k-1)-y_{2, j}(k) y_{j}(k-1)-y_{2, j}(k-1) y_{j}(k)\right) \\
& -\frac{2 F_{j}}{\lambda_{j}^{2}} \sum_{k=-N}^{N} \delta a_{k} 2 \varphi_{j}(k) \varphi_{j}(k-1) .
\end{aligned}
$$

Аналогично $\left\{\lambda_{i}, \lambda_{j}\right\}$ вычислим $\left\{\lambda_{i}, 2 F_{j} / \lambda_{j}\right\}$, учитьвая при этом, что $y_{2, j}$ и $y_{j}-$ peшения Флоке с взаимнообратными множителями, $\varphi_{i}(-N)=\varphi_{i}(N+1)=0$. Заметим, что члены при $Z_{j}$ и $F_{j}$ равны 0 , так как они пропорциональны $\left\{\lambda_{i}, \lambda_{j}\right\}$. Опустив их, получим

$$
\begin{aligned}
\left\{\lambda_{i}, \frac{2 F_{j}}{\lambda_{j}}\right\}= & \frac{1}{a_{N+2} \lambda_{j}} \sum_{k=-N}^{N}\left(\left(y_{2, j}(k) y_{j}(k-1)+y_{j}(k) y_{2, j}(k-1)\right) a_{k} a_{k+1} \varphi_{i}(k) \varphi_{i}(k+1)\right. \\
& \left.-\left(y_{2, j}(k+1) y_{j}(k)+y_{j}(k+1) y_{2, j}(k)\right) a_{k} a_{k+1} \varphi_{i}(k) \varphi_{i}(k-1)\right)
\end{aligned}
$$

Рассмотрим случай $i=j$. В этом случае после раскрытия скобок два слагаемых взаимно уничтожатся. Выберем $C$ таким, что $y_{2}=C \varphi$, и, опустив индексы $i, j$, получим

$$
\frac{C}{a_{N+2} \lambda} \sum_{k=-N}^{N} a_{k} a_{k+1}(\varphi(k) y(k-1) \varphi(k+1) \varphi(k)-\varphi(k) y(k+1) \varphi(k) \varphi(k-1)) .
$$

Проделав преобразование $a_{k+1} \varphi(k+1)=\lambda \varphi(k)-a_{k} \varphi(k-1)$ и аналогичное преобразование для $a_{k+1} y(k+1)$, получим

$$
\begin{aligned}
\frac{C}{a_{N+2}} \sum_{k=-N}^{N} \varphi^{2}(k)\left(a_{k} y(k-1) \varphi(k)-a_{k} \varphi(k-1) y(k)\right) & =\frac{C}{a_{N+2}} \sum_{k=-N}^{N} \varphi^{2}(k) W_{k-1}(y, \varphi) \\
& =\sum_{k=-N}^{N} \varphi^{2}(k)=1,
\end{aligned}
$$

так как $W_{k-1}(y, \varphi)=a_{-N+1} / C$ и $a_{-N+1}=a_{N+2}$.

В случае $i \neq j$ с помощью аналогичных выкладок можно получить, что $\left\{\lambda_{i}, 2 F_{j} / \lambda_{j}\right\}$ с точностью до множителей равно

$$
\begin{gathered}
\sum_{k=-N}^{N}\left(\left(W_{k+1}^{\uparrow}\left(y_{2, j}, \varphi_{i}\right)-W_{k-1}^{\uparrow}\left(y_{2, j}, \varphi_{i}\right)\right) W_{k-1}^{\downarrow}\left(y_{j}, \varphi_{i}\right)\right. \\
\left.\quad+\left(W_{k+1}^{\uparrow}\left(y_{j}, \varphi_{i}\right)-W_{k-1}^{\uparrow}\left(y_{j}, \varphi_{i}\right)\right) W_{k-1}^{\downarrow}\left(y_{2, j}, \varphi_{i}\right)\right),
\end{gathered}
$$

что равно 0 в силу леммы 1 и равенства $W_{k}^{\uparrow}\left(y_{2, j}, \varphi_{i}\right)=0$ при $k=-N-1,-N, N+1, N$.

Аналогичным образом доказывается и равенство $\left\{2 F_{i} / \lambda_{i}, 2 F_{j} / \lambda_{j}\right\}=0$. 
Теорема 2. Координаты $\left(\lambda_{i}, 2 F_{j} / \lambda_{j}^{3}: i, j=1, \ldots, N\right)$ являются канонически сопряженными для кубической скобки, т.е.

$$
\left\{\lambda_{i}, \lambda_{j}\right\}_{2}=0, \quad\left\{\lambda_{i}, \frac{2 F_{j}}{\lambda_{j}^{3}}\right\}_{2}=\delta_{i, j}, \quad\left\{\frac{2 F_{i}}{\lambda_{i}^{3}}, \frac{2 F_{j}}{\lambda_{j}^{3}}\right\}_{2}=0
$$

ЗАМЕчАниЕ. Как и в случае квадратичной скобки, фазовое пространство в нечетном случае имеет размерность $2 N+1$, но далее будет показано, что размерность аннулятора кубической скобки равна 1 (соответственно $2 N+2$ и 2 в четном случае), поэтому это полньй набор канонически сопряженных переменных.

Доказательство будет проведено для нечетного случая, так как в четном все аналогично.

Лемма 3. Пусть последовательности $A_{k}, B_{k}, Q_{k}$ таковь, ито $A_{k}+A_{k-1}=$ $B_{k}-B_{k-1}, Q_{k}=\left(A_{k}+B_{k}\right)^{2} / 4$. Тогдa

$$
\sum_{k=-N}^{N} A_{k} B_{k}=Q_{N}-Q_{-N-1}
$$

ДокаЗАТЕЛЬСтво. Легко проверить, что $Q_{k}-Q_{k-1}=A_{k} B_{k}$, откуда немедленно следует утверждение леммы.

Лемма 4. Пусть $\psi_{i}, \psi_{j}$ - произвольные решения уравнения $l \psi_{i}=\lambda_{i} \psi_{i}, i=1,2$. $\Pi y c b_{b} W_{k}=W_{k}\left(\psi_{i}, \psi_{j}\right) u$

$$
W_{k}^{+}=W_{k}^{+}\left(\psi_{i}, \psi_{j}\right)=a_{k+1}\left(\psi_{i}(k) \psi_{j}(k+1)+\psi_{i}(k+1) \psi_{j}(k)\right) .
$$

Пусть

$$
\mathscr{W}\left(\psi_{i}, \psi_{j}\right)=\left(\frac{W_{N}^{+}}{\lambda_{j}+\lambda_{i}}+\frac{W_{N}}{\lambda_{j}-\lambda_{i}}\right)^{2}-\left(\frac{W_{-N-1}^{+}}{\lambda_{j}+\lambda_{i}}+\frac{W_{-N-1}}{\lambda_{j}-\lambda_{i}}\right)^{2}
$$

Тогда

$$
\sum_{k=-N}^{N} W_{k}^{+} W_{k}=\frac{1}{4}\left(\lambda_{j}^{2}-\lambda_{i}^{2}\right) \mathscr{W}\left(\psi_{i}, \psi_{j}\right)
$$

ДоКАЗАТЕЛЬСТво немедленно следует из леммы 3 , если заметить, что

$$
W_{k}^{+}+W_{k-1}^{+}=\left(\lambda_{j}+\lambda_{i}\right) \psi_{i}(k) \psi_{j}(k), \quad W_{k}-W_{k-1}=\left(\lambda_{j}-\lambda_{i}\right) \psi_{i}(k) \psi_{j}(k)
$$


ДоКАЗАТЕЛЬСтво ТЕоРЕмЫ 2. Так как в данном доказательстве упоминается только кубическая скобка $\{\cdot, \cdot\}_{2}$, обозначим ее для простоты через $\{\cdot, \cdot\}$.

Аналогично доказательству теоремы 1 можно найти, что $\left\{\lambda_{i}, \lambda_{j}\right\}$ равно

$$
\begin{aligned}
& \sum_{k=-N}^{N} a_{k} a_{k+1}\left(a_{k}^{2}+a_{k+1}^{2}\right)\left(\varphi_{i}(k) \varphi_{i}(k-1) \varphi_{j}(k+1) \varphi_{j}(k)\right. \\
& \left.\quad-\varphi_{i}(k+1) \varphi_{i}(k) \varphi_{j}(k) \varphi_{j}(k-1)\right)+a_{k} a_{k+1}^{2} a_{k+2}\left(\varphi_{i}(k) \varphi_{i}(k-1) \varphi_{j}(k+2) \varphi_{j}(k+1)\right. \\
& \left.\quad-\varphi_{i}(k+2) \varphi_{i}(k+1) \varphi_{j}(k) \varphi_{j}(k-1)\right) .
\end{aligned}
$$

Послепроведения во вторых кругльх скобках преобразования $a_{k+2} \varphi(k+2)=\lambda \varphi(k+1)-$ $a_{k+1} \varphi(k)$, сократив в обоих скобках взаимно уничтожаюшиеся члены, получим

$$
\begin{aligned}
& \sum_{k=-N}^{N} a_{k}^{3} a_{k+1}\left(\varphi_{i}(k) \varphi_{i}(k-1) \varphi_{j}(k+1) \varphi_{j}(k)-\varphi_{i}(k+1) \varphi_{i}(k) \varphi_{j}(k) \varphi_{j}(k-1)\right) \\
& +a_{k} a_{k+1}^{2}\left(\varphi_{i}(k) \varphi_{i}(k-1) \lambda_{j} \varphi_{j}(k+1) \varphi_{j}(k+1)-\varphi_{i}(k+1) \varphi_{i}(k+1) \lambda_{i} \varphi_{j}(k) \varphi_{j}(k-1)\right) .
\end{aligned}
$$

Проведем теперь в первых круглых скобках преобразование $a_{k} \varphi(k-1)=\lambda \varphi(k)-$ $a_{k+1} \varphi(k+1)$ :

$$
\begin{aligned}
& \sum_{k=-N}^{N} a_{k}^{2} a_{k+1}\left(\varphi_{i}(k) \varphi_{i}(k) \lambda_{i} \varphi_{j}(k+1) \varphi_{j}(k)-\varphi_{i}(k) \varphi_{i}(k+1) \lambda_{j} \varphi_{j}(k) \varphi_{j}(k)\right) \\
& +a_{k} a_{k+1}^{2}\left(\varphi_{i}(k) \varphi_{i}(k-1) \lambda_{j} \varphi_{j}(k+1) \varphi_{j}(k+1)-\varphi_{i}(k+1) \varphi_{i}(k+1) \lambda_{i} \varphi_{j}(k) \varphi_{j}(k-1)\right) .
\end{aligned}
$$

Проделаем теперь в первых круглых скобках преобразование $a_{k+1} \varphi(k+1)=\lambda \varphi(k)-$ $a_{k} \varphi(k-1)$, а во вторых $a_{k} \varphi(k-1)=\lambda \varphi(k)-a_{k+1} \varphi(k+1)$ :

$$
\begin{aligned}
& \sum_{k=-N}^{N} a_{k}^{3}\left(\varphi_{i}(k) \varphi_{i}(k-1) \lambda_{j} \varphi_{j}(k) \varphi_{j}(k)-\varphi_{i}(k) \varphi_{i}(k) \lambda_{i} \varphi_{j}(k-1) \varphi_{j}(k)\right) \\
& \quad+a_{k+1}^{2}\left(\varphi_{i}(k) \varphi_{i}(k) \lambda_{i} \lambda_{j} \varphi_{j}(k+1) \varphi_{j}(k+1)-\varphi_{i}(k+1) \varphi_{i}(k+1) \lambda_{i} \lambda_{j} \varphi_{j}(k) \varphi_{j}(k)\right) \\
& \quad-a_{k+1}^{3}\left(\varphi_{i}(k) \varphi_{i}(k+1) \lambda_{j} \varphi_{j}(k+1) \varphi_{j}(k+1)-\varphi_{i}(k+1) \varphi_{i}(k+1) \lambda_{i} \varphi_{j}(k) \varphi_{j}(k+1)\right)
\end{aligned}
$$

В последних круглых скобках сделаем замену индекса суммирования $k+1 \mapsto k^{\prime}$, после чего видно, что первые и третьи круглые скобки в сумме дают 0 .

Преобразуем оставшееся выражение и воспользуемся леммой 4:

$$
\begin{aligned}
& \sum_{k=-N}^{N} a_{k+1}^{2}\left(\varphi_{i}^{2}(k) \lambda_{i} \lambda_{j} \varphi_{j}^{2}(k+1)-\varphi_{i}^{2}(k+1) \lambda_{i} \lambda_{j} \varphi_{j}^{2}(k)\right) \\
& \quad=\sum_{k=-N}^{N} \lambda_{i} \lambda_{j} W_{k}^{+}\left(\varphi_{i}, \varphi_{j}\right) W_{k}\left(\varphi_{i}, \varphi_{j}\right)=\frac{1}{4} \lambda_{i} \lambda_{j}\left(\lambda_{j}^{2}-\lambda_{i}^{2}\right) \mathscr{W}\left(\varphi_{i}, \varphi_{j}\right) .
\end{aligned}
$$

Но из того, что $\varphi(-N)=\varphi(N+1)=0$, следует $\mathscr{W}\left(\varphi_{i}, \varphi_{j}\right)=0$, откуда получаем первое утверждение теоремы. 
Аналогично доказательству теоремы 1 можно вычислить $\left\{\lambda_{i}, 2 F_{j} / \lambda_{j}\right\}$. При этом получим следующее выражение:

$$
\begin{aligned}
& \frac{1}{a_{N+2} \lambda_{j}^{3}} \sum_{k=-N}^{N} a_{k} a_{k+1}\left(a_{k}^{2}+a_{k+1}^{2}\right)\left(\left(y_{2, j}(k) y_{j}(k-1)+y_{2, j}(k-1) y_{j}(k)\right) \varphi_{i}(k+1) \varphi_{i}(k)\right. \\
& \left.\quad-\left(y_{2, j}(k+1) y_{j}(k)+y_{2, j}(k) y_{j}(k+1)\right) \varphi_{i}(k) \varphi_{i}(k-1)\right) \\
& \quad+a_{k} a_{k+1}^{2} a_{k+2}\left(\left(y_{2, j}(k) y_{j}(k-1)+y_{2, j}(k-1) y_{j}(k)\right) \varphi_{i}(k+2) \varphi_{i}(k+1)\right. \\
& \left.\quad-\left(y_{2, j}(k+2) y_{j}(k+1)+y_{2, j}(k+1) y_{j}(k+2)\right) \varphi_{i}(k) \varphi_{i}(k-1)\right)
\end{aligned}
$$

Проделав преобразования, аналогичные преобразованиям при доказательстве равенства $\left\{\lambda_{i}, \lambda_{j}\right\}=0$, получим, что последнее выражение равно

$$
\frac{1}{a_{N+2} \lambda_{j}^{3}} \sum_{k=-N}^{N} 2 a_{k+1}^{2}\left(y_{2, j}(k) y_{j}(k) \lambda_{i} \lambda_{j} \varphi_{i}^{2}(k+1)-y_{2, j}(k+1) y_{j}(k+1) \lambda_{i} \lambda_{j} \varphi_{i}^{2}(k)\right) .
$$

Рассмотрим случай $i=j$. Опустим индексы $i, j$. Пусть $C$ таково, что $y_{2}=C \varphi$. Тогда находим

$$
\frac{2 C}{a_{N+2} \lambda} \sum_{k=-N}^{N} a_{k+1}^{2}\left(\varphi(k) y(k) \varphi^{2}(k+1)-\varphi(k+1) y(k+1) \varphi^{2}(k)\right) .
$$

Проделаем преобразование $a_{k+1} \varphi(k+1)=\lambda \varphi(k)-a_{k} \varphi(k-1)$ :

$$
\begin{aligned}
& \frac{2 C}{a_{N+2} \lambda} \sum_{k=-N}^{N} a_{k+1} \lambda \varphi^{2}(k)(y(k) \varphi(k+1)-y(k+1) \varphi(k)) \\
& \quad+a_{k} \varphi(k) \varphi(k-1) a_{k+1}(y(k+1) \varphi(k)-y(k) \varphi(k+1)) \\
& =\frac{2 C}{a_{N+2} \lambda} \sum_{k=-N}^{N} \lambda \varphi^{2}(k) W_{k}(y, \varphi)-a_{k} \varphi(k) \varphi(k-1) W_{k}(y, \varphi) .
\end{aligned}
$$

Но $W_{k}(y, \varphi)=W_{-N}(y, \varphi)=a_{N+2} / C$, откуда

$$
\left\{\lambda_{i}, \frac{2 F_{i}}{\lambda_{i}^{3}}\right\}=2 \sum_{k=-N}^{N} \varphi^{2}(k)-\frac{2}{\lambda} \sum_{k=-N}^{N} a_{k} \varphi(k) \varphi(k-1) .
$$

Первое слагаемое равно 2 в силу нормировки $\varphi$. Рассмотрим второе слагаемое. Сделаем в нем преобразование $a_{k} \varphi(k-1)=\lambda \varphi(k)-a_{k+1} \varphi(k+1)$ :

$$
\frac{1}{\lambda} \sum_{k=-N}^{N} a_{k} \varphi(k) \varphi(k-1)=\sum_{k=-N}^{N} \varphi^{2}(k)-\frac{1}{\lambda} \sum_{k=-N}^{N} a_{k+1} \varphi(k+1) \varphi(k) .
$$

Во втором слагаемом можно сделать замену индекса $k+1 \mapsto k^{\prime}$ и из полученного выражения можно найти, что

$$
\frac{1}{\lambda} \sum_{k=-N}^{N} a_{k} \varphi(k) \varphi(k-1)=\frac{1}{2}
$$


откуда следует, что $\left\{\lambda_{i}, 2 F_{i} / \lambda_{i}^{3}\right\}=1$.

Рассмотрим теперь случай $i \neq j$. Заменив $y_{2, j}(k) y_{j}(k)$ в выражении для $\left\{\lambda_{i}, 2 F_{j} / \lambda_{j}^{3}\right\}$ выражением $\left(\left(y_{2, j}(k)+y_{j}(k)\right)^{2}-y_{2, j}^{2}(k)-y_{j}^{2}(k)\right) / 2$, получим

$$
\begin{aligned}
& \frac{\lambda_{i}}{a_{N+2} \lambda_{j}^{2}} \sum_{k=-N}^{N} a_{k+1}^{2}\left(\left(y_{2, j}(k)+y_{j}(k)\right)^{2} \varphi_{i}^{2}(k+1)-\left(y_{2, j}(k+1)+y_{j}(k+1)\right)^{2} \varphi_{i}^{2}(k)\right) \\
& \quad-a_{k+1}^{2}\left(y_{2, j}(k)^{2} \varphi_{i}^{2}(k+1)-y_{2, j}(k+1)^{2} \varphi_{i}^{2}(k)\right) \\
& \quad-a_{k+1}^{2}\left(y_{j}(k)^{2} \varphi_{i}^{2}(k+1)-y_{j}(k+1)^{2} \varphi_{i}^{2}(k)\right) .
\end{aligned}
$$

После этого можно, как и раньше, воспользоваться леммой 4. С точностью до множителя мы получим

$$
\mathscr{W}\left(y_{2, j}+y_{j}, \varphi_{i}\right)-\mathscr{W}\left(y_{2, j}, \varphi_{i}\right)-\mathscr{W}\left(y_{j}, \varphi_{i}\right)
$$

Учитьвая определения $\varphi, y, y_{2}$, можно непосредственным вычислением убедиться, что это выражение равно 0.

Доказательство того, что $\left\{2 F_{i} / \lambda_{i}^{3}, 2 F_{j} / \lambda_{j}^{3}\right\}=0$, проводится аналогично.

ЗАМЕЧАНИЕ. Из определения $F_{i}$ видно, что если перейти от $\lambda_{i} \mathrm{~K}-\lambda_{i}$ и соответственно изменить $F_{i}$, то результаты теорем 1 и 2 не изменятся.

Рассмотрим теперь $\Delta(\lambda)$ - след матрицы монодромии $U$. Пусть $\Pi=\prod_{i=-N}^{N} a_{i}$ в нечетном случае и $\Pi=\prod_{i=-N}^{N+1} a_{i}$ в четном. Определим величины $J_{i}$ следующим образом: при натуральных $i$ это сумма всевозможных произведений $i$ штук различных $a_{k}^{2}$, в которых не встречаются $a$ с соседними номерами (с учетом периодичности), а $J_{0}=1$.

ЛЕмма 5. Выполнено

$$
\Delta(\lambda)=\left\{\begin{array}{l}
\sum_{i=0}^{N}(-1)^{i} \lambda^{2 N+1-2 i} \frac{J_{i}}{\Pi}, \quad \text { в нечетном случае } \\
\sum_{i=0}^{N+1}(-1)^{i} \lambda^{2 N+2-2 i} \frac{J_{i}}{\Pi} . \quad \text { в четном случае }
\end{array}\right.
$$

ДокаЗАТЕЛЬСтво. Рассмотрим $U$ в базисе $y_{1}, y_{2}$. В нечетном случае, например, $\operatorname{tr} U=y_{1}(N+1)+y_{2}(N+2)$. Пользуясь уравнением $l y_{k}=\lambda y_{k}, k=1,2$, как рекуррентным соотношением, можно найти $y_{1}(N+1), y_{2}(N+2)$ и получить утверждение леммы.

Пусть $I_{k}=J_{k} / \Pi$.

ЗАмЕчаниЕ. Легко убедиться, что $I_{k}=0$ при $k \geqslant N+1$ в нечетном случае и $k \geqslant N+2$ в четном.

Теорема 3. Виражсения $I_{k}$ удовлетворяют соотношениям $\left\{I_{0}, f\right\}_{1}=0,\left\{I_{k}, f\right\}_{2}$ $=-\left\{I_{k+1}, f\right\}_{1},\left\{I_{N}, f\right\}_{2}=0$, а в четном случае $\left\{I_{N+1}, f\right\}_{2}=\left\{I_{N+1}, f\right\}_{1}=0$, где $f$ - произвольная функиия. 
ДокАЗАТЕЛЬСТво. Введем величины $\check{J}_{k}(n)$ и $\hat{J}_{k}(n)$ следующим образом: $\check{J}_{k}(n)$ равно выражению $J_{k}$, из которого выкинуты все слагаемые, содержащие $a_{n}, a_{n \pm 1}$ (с учетом периодичности); $\hat{J}_{k}(n)$ равно выражению $J_{k}$, из которого выкинуты все слагаемые, содержашие $a_{n}, a_{n \pm 1}, a_{n \pm 2}$ (с учетом периодичности). Ясно, что $\partial J_{k} / \partial a_{n}=2 a_{n} \breve{J}_{k-1}(n)$, причем для того чтобы это равенство сохранялось и при $k=0,1$, положим $\breve{J}_{-1}(n)=0$, $\breve{J}_{0}(n)=1$. Отметим также тождество

$$
J_{k}-\check{J}_{k}(n)=a_{n}^{2} \check{J}_{k-1}(n)+a_{n-1}^{2} \check{J}_{k-1}(n-1)+a_{n+1}^{2} \check{J}_{k-1}(n+1)-a_{n-1}^{2} a_{n+1}^{2} \hat{J}_{k-2}(n),
$$

причем для того чтобы это равенство сохранялось и при $k=0,1,2$, положим $\hat{J}_{-2}(n)=0$, $\hat{J}_{-1}(n)=0, \hat{J}_{0}(n)=1$. Отметим также тождество $\partial \Pi / \partial a_{n}=\Pi / a_{n}$.

Теорему достаточно доказать для $f=c_{n}=a_{n}^{2}$. Докажем первое утверждение:

$$
\left\{\frac{J_{0}}{\Pi}, c_{n}\right\}_{1}=\left\{\frac{1}{\Pi}, c_{n}\right\}_{1}=-\frac{1}{\Pi^{2}} \frac{\Pi}{2}\left(a_{n}^{2}-a_{n}^{2}\right)=0 .
$$

Перейдем ко второму утверждению. Можно подсчитать, что

$$
\begin{aligned}
& -\left\{\frac{J_{k+1}}{\Pi}, c_{n}\right\}_{1}=-\frac{a_{n}^{2}}{\Pi}\left(a_{n-1}^{2} \check{J}_{k}(n-1)-a_{n+1}^{2} \check{J}_{k}(n+1)\right), \\
\left\{\frac{J_{k}}{\Pi}, c_{n}\right\}_{2}= & \frac{a_{n}^{2}}{\Pi} J_{k}\left(a_{n+1}^{2}-a_{n-1}^{2}\right)+\frac{a_{n}^{2}}{\Pi}\left(\check{J}_{k-1}(n-1) a_{n-1}^{2}\left(a_{n-1}^{2}+a_{n}^{2}\right)\right. \\
& -\check{J}_{k-1}(n+1) a_{n+1}^{2}\left(a_{n+1}^{2}+a_{n}^{2}\right)+\check{J}_{k-1}(n-2) a_{n-2}^{2} a_{n-1}^{2} \\
& \left.-\check{J}_{k-1}(n+2) a_{n+2}^{2} a_{n+1}^{2}\right) \\
= & \frac{a_{n}^{2}}{\Pi}\left(a_{n+1}^{2}\left(J_{k}-\check{J}_{k-1}(n+1) a_{n+1}^{2}-\check{J}_{k-1}(n+1) a_{n}^{2}-\check{J}_{k-1}(n+2) a_{n+2}^{2}\right)\right. \\
& \left.-a_{n-1}^{2}\left(J_{k}-\check{J}_{k-1}(n-1) a_{n-1}^{2}-\check{J}_{k-1}(n-1) a_{n}^{2}-\check{J}_{k-1}(n-2) a_{n-2}^{2}\right)\right) .
\end{aligned}
$$

Воспользовавшись тождеством (4) при $n \pm 1$, получим

$$
\begin{aligned}
\left\{\frac{J_{k}}{\Pi}, c_{n}\right\}_{2}= & \frac{a_{n}^{2}}{\Pi}\left(a_{n+1}^{2}\left(\check{J}_{k}(n+1)-\check{J}_{k-1}(n+1) a_{n}^{2}+\check{J}_{k-1}(n) a_{n}^{2}-\hat{J}_{k-2}(n+1) a_{n}^{2} a_{n+2}^{2}\right)\right. \\
& \left.-a_{n-1}^{2}\left(\check{J}_{k}(n-1)-\check{J}_{k-1}(n-1) a_{n}^{2}+\check{J}_{k-1}(n) a_{n}^{2}-\hat{J}_{k-2}(n-1) a_{n}^{2} a_{n-2}^{2}\right)\right) .
\end{aligned}
$$

Воспользовавшись равенством (5) и тождествами

$$
\check{J}_{k-1}(n \mp 1)-\check{J}_{k-1}(n)=a_{n \pm 1}^{2} \hat{J}_{k-2}(n)-a_{n \mp 2}^{2} \hat{J}_{k-2}(n \mp 1),
$$

получим

$$
\begin{aligned}
- & \left\{\frac{J_{k+1}}{\Pi}, c_{n}\right\}_{1}+\frac{a_{n}^{2}}{\Pi}\left(a_{n}^{2} a_{n+1}^{2}\left(-a_{n-1}^{2} \hat{J}_{k-2}(n)\right)-a_{n}^{2} a_{n-1}^{2}\left(-a_{n+1}^{2} \hat{J}_{k-2}(n)\right)\right) \\
& =-\left\{\frac{J_{k+1}}{\Pi}, c_{n}\right\}_{1} .
\end{aligned}
$$

Таким образом, второе утверждение теоремы доказано. Заметим, что оно верно даже при $J_{k}$ или $J_{k+1}$ равных 0. 
Рассмотрим четвертое утверждение теоремы. Заметим, что

$$
\begin{gathered}
\frac{J_{N+1}}{\Pi}=\frac{\Pi_{1}}{\Pi_{2}}+\frac{\Pi_{2}}{\Pi_{1}}, \\
\Pi_{1}=a_{-N} a_{-N+2} \cdots a_{N-2} a_{N}, \quad \Pi_{2}=a_{-N+1} a_{-N+3} \cdots a_{N-1} a_{N+1} .
\end{gathered}
$$

Непосредственным вычислением можно проверить, что для любой функции $f$ справедливы равенства $\left\{\Pi_{1}, f\right\}_{1}=\left\{\Pi_{2}, f\right\}_{1}=0,\left\{\Pi_{1} / \Pi_{2}, f\right\}_{2}=0$, откуда следует четвертое утверждение леммы.

В четном случае третье утверждение следует из четвертого, а в нечетном из того, что $J_{N+1}=0$.

СлЕДСТВИЕ 1. В нечетном случае аннулятор квадратичной скобки порождается функиией $I_{0}$, а кубической - функиией $I_{N}$, поэтому обе скобки однократно вырождены. В четном случае аннулятор квадратичной скобки порождается функииями $I_{N+1}$ и $I_{0}$, а аннулятор кубической скобки порожсдется функииями $I_{N+1}$ и $I_{N}$, поэтому обе скобки двукратно вырождены.

ДокАЗАТЕЛЬСТво. В нечетном случае утверждение следует из теоремы 3. В четном случае можно заметить, что функция $I_{N+1}$ независима от функций $I_{N}$ и $I_{0}$, после чего утверждение также следует из теоремы 3.

СлЕДСТВИЕ 2. Функиии $I_{k}$ находятся в инволюции относительно обеих скобок.

ДоКАЗАТЕЛЬСТВо состоит в прямом вычислении:

$$
\begin{aligned}
\left\{I_{k}, I_{l}\right\}_{1} & =-\left\{I_{k}, I_{l-1}\right\}_{2}=\left\{I_{l-1}, I_{k}\right\}_{2}=-\left\{I_{l-1}, I_{k+1}\right\}_{1} \\
& =\left\{I_{k+1}, I_{l-1}\right\}_{1}=\cdots=\left\{I_{k+l}, I_{0}\right\}_{1}=0 .
\end{aligned}
$$

СлЕДСТВИЕ 3. Интегралы $I_{0}, \ldots, I_{N}$ совпадают с интегралами, получаемыми процедурой Магри [10] для пары скобок Пуассона $\{\cdot, \cdot\}_{1},\{\cdot, \cdot\}_{2}$.

ЛЕмма 6. Поток, порождаемый $I_{1}$ с помощью квадратичной скобки, на ее симплектических листах совпадает с точностью до мнохителя с потоком Вольтерра.

ДокАЗАТЕЛЬСТво. Симплектические листы задаются уравнениями $1 / \Pi=C$ в нечетном случае и $1 / \Pi=C, J_{N+1} / \Pi=C_{1}$ в четном. Поток с гамильтонианом $I_{1}$ имеет вид

$$
\dot{c}_{k}=\left\{\frac{J_{1}}{\Pi}, c_{k}\right\}_{1}=\frac{c_{k}^{2}}{\Pi}\left(c_{k-1}^{2}-c_{k+1}^{2}\right)=-C c_{k}^{2}\left(c_{k+1}^{2}-c_{k-1}^{2}\right),
$$

откуда следует утверждение леммы.

Лемма 6 объясняет интерес к потокам, порождаемым $I_{k}$. Заметим, что согласно лемме 6 и следствию 2 из леммы 5 имеет место интегрируемость системы Вольтерра. Рассмотрим потоки, порождаемые интегралами $I_{1}, \ldots, I_{N}$ с помошью квадратичной скобки и, что то же самое, интегралами $-I_{0}, \ldots,-I_{N-1}$ с помощью кубической скобки.

Заметим, что участвующий в определении $F_{k}=\ln \left(\left|\rho_{k}\right|\right)$ модуль необходим только для вещественности $F_{k}$, и его можно опустить без потери каких-либо результатов. Опустим его для простоты выкладок и будем использовать для квадратичной скобки 
канонически сопряженные переменные $\left( \pm \mathscr{F}{ }_{k}=\left(2 \ln \rho_{k}\right) / \lambda_{k}, \lambda_{k}\right)$, а для кубической скобки канонически сопряженные переменные $\left( \pm \mathscr{G}_{k}=\left(2 \ln \rho_{k}\right) / \lambda_{k}^{3}, \lambda_{k}\right)$. Знак \pm возникает от неоднозначности выбора между $\rho_{k}$ и $1 / \rho_{k}$ и зависит от того, на каком листе лежит соответствующий $\lambda_{k}$ полюс $P_{k}$.

Известно, что производящая функция канонического преобразования $p, q \mapsto I, \varphi$ перехода к переменным “действие-угол” $I, \varphi$ вьглядит так:

$$
S(I, q)=\int_{q_{0}}^{q} p(I, q) d q
$$

причем $\varphi=\partial S(I, q) / \partial I$. Мы же воспользуемся не переменными “действие", а инволютивными интегралами $I_{k}$ :

$$
S\left(I_{1}, \ldots, I_{N}, \lambda_{1}, \ldots, \lambda_{N}\right)=\int_{\lambda_{1,0}, \ldots, \lambda_{N, 0}}^{\lambda_{1}, \ldots, \lambda_{N}} \sum_{k=1}^{N} \pm \mathscr{F}_{k} d \lambda_{k}
$$

для квадратичной скобки и, аналогично,

$$
S\left(-I_{0}, \ldots,-I_{N-1}, \lambda_{1}, \ldots, \lambda_{N}\right)=\int_{\lambda_{1,0}, \ldots, \lambda_{N, 0}}^{\lambda_{1}, \ldots, \lambda_{N}} \sum_{k=1}^{N} \pm \mathscr{G}_{k} d \lambda_{k}
$$

для кубической скобки; при этом функции $\partial S / \partial I_{k}$ для квадратичной скобки и $\partial S / \partial\left(-I_{k}\right)$ для кубической скобки задают координаты на торе Лиувилля, отличающиеся от переменных “угол” на аффинное преобразование. Рассмотрим на кривой Г базис голоморфных дифференциалов, инвариантных относительно инволюции $\sigma$ следующего вида:

$$
\omega_{k}=\left\{\begin{array}{ll}
\frac{\lambda^{2 k+1} d \lambda}{y} & \text { в четном случае, } \\
\frac{\lambda^{2 k} d \lambda}{y} & \text { в нечетном случае }
\end{array} \quad k=0, \ldots, N-1 .\right.
$$

Пусть $Q_{1}, \ldots, Q_{N}-$ набор точек на $\Gamma$. Определим модифицированное отображение Абеля $A: S^{N} \Gamma \rightarrow J(\Gamma / \sigma)$ следующим образом:

$$
A^{k}\left(Q_{1}, \ldots, Q_{N}\right)=\sum_{j=1}^{N} \int_{P}^{Q_{j}} \omega_{k}, \quad k=0, \ldots, N-1,
$$

где $P$ - некая фиксированная точка $\Gamma$.

ТЕорема 4. Координаты $\partial S / \partial I_{k}$ для квадратичной скобки и $\partial S / \partial\left(-I_{k}\right)$ для кубической скобки представляют собой с точностью до знака модифичированное отобрахение Абеля от $\left(P_{1}, \ldots, P_{N}\right)$ :

$$
\frac{\partial S}{\partial I_{k}}=(-1)^{k} A^{N-k}\left(P_{1}, \ldots, P_{N}\right)=\sum_{j=1}^{N} \int_{P}^{P_{j}}(-1)^{k} \omega_{N-k}, \quad k=1, \ldots, N,
$$

для квадратичной скобки и

$$
\begin{gathered}
\frac{\partial S}{\partial\left(-I_{k}\right)}=(-1)^{k-1} A^{N-k-1}\left(P_{1}, \ldots, P_{N}\right)=\sum_{j=1}^{N} \int_{P}^{P_{j}}(-1)^{k-1} \omega_{N-k-1}, \\
k=0, \ldots, N-1,
\end{gathered}
$$

для кубической скобки, әде $P$ - некая фиксированная точка, а $P_{j}$ - вышеописанные полюсы $\psi(n)$. 
ДокАЗАТЕЛЬСтво. Рассмотрим только случай квадратичной скобки при нечетном периоде (остальные случаи аналогичны):

$$
\begin{aligned}
\frac{\partial S}{\partial I_{k}} & =\left.\frac{\partial}{\partial I_{k}}\left(\sum_{j=1}^{N} \int_{\lambda_{j, 0}}^{\lambda_{j}} \pm \mathscr{F}_{j} d \lambda_{j}\right)\right|_{I_{0}=\mathrm{const}} \\
& =\sum_{j=1}^{N} \int_{\lambda_{j, 0}}^{\lambda_{j}} \frac{2}{\lambda_{j}} \frac{\partial}{\partial I_{k}} \ln \left(\frac{\Delta\left(\lambda_{j}\right)}{2} \pm\left.\sqrt{\left.\frac{\left(\Delta\left(\lambda_{j}\right)\right)^{2}}{4}-1\right)} d \lambda_{j}\right|_{I_{0}=\mathrm{const}}\right. \\
& =\left.\sum_{j=1}^{N} \int_{\lambda_{j, 0}}^{\lambda_{j}} \frac{2}{\lambda_{j}} \frac{1}{ \pm \sqrt{\left(\Delta\left(\lambda_{j}\right)\right)^{2} / 4-1}} \frac{\partial\left(\lambda_{j}\right)}{\partial I_{k}} d \lambda_{j}\right|_{I_{0}=\mathrm{const}} ^{N}(-1)^{k} \lambda_{j}^{2 N+1-2 k} d \lambda_{j}=\sum_{j=1}^{\lambda_{j}} \int_{\lambda_{j, 0}}(-1)^{k} \frac{\lambda_{j}^{2(N-k)}}{y} d \lambda_{j}, \\
& =\sum_{j=1}^{\lambda_{j}} \int_{\lambda_{j, 0}}^{\frac{1}{\lambda_{j}}} \frac{1}{ \pm \sqrt{R\left(\lambda_{j}\right)}}
\end{aligned}
$$

где знак плюс или минус соответствует листу, на котором лежит $P_{j}$.

Из полученного выражения, использовав лемму 5 , легко получить требуемое утверждение.

ЗАмечаниЕ. Легко заметить, что если заменить $\lambda_{j}$ на $-\lambda_{j}$ и соответственно $P_{j}$ на $P_{N+j}=\sigma\left(P_{j}\right)$, то результаты теоремы 4 не изменятся.

Автор хотел бывыразить свою признательность А. П. Веселову за постановку задачи и полезные обсуждения.

\section{СПИСОК ЦИТИРОВАННОЙ ЛИТЕРАТУРЫ}

[1] Тахтаджян Л. А., Фаддеев Л. Д. Гамильонов подход в теории солитонов. М.: Наука, 1986.

[2] Damianou P. A. The Volterra model and its relation to the Toda lattice // Phys. Lett. A. 1991. V. 155. № 2-3. P. 126-132.

[3] Манаков С. В. О полной интегрируемости и стохастизации в дискретных динамических системах // ЖЭТФ. 1974. Т. 67. № 2. С. 543-555.

[4] Kac M., van Moerbeke P. On an explicitly soluble system of nonlinear differential equations related to certain Toda lattices // Adv. Math. 1975. V. 16. P. 160-169.

[5] Flaschka H., McLaughlin D. W. Canonically conjugate variables for the Korteweg-de Vries equation and the Toda lattice with periodic boundary conditions // Progr. Theoret. Phys. 1976. V. 55. № 2. P. 438-456.

[6] Верещагин В. Л. Спектральная теория однофазных решений цепочки Вольтерра // Матем. заметки. 1990. Т. 48. №2. С. 145-148.

[7] Кричевер И. М. Нелинейные уравнения и эллиптические кривые // Итоги науки и техн. Соврем. пробл. матем. Фундамент. направления. Т. 23. М.: ВИНИТИ, 1983. С. 79-136.

[8] Дубровин Б. А., Матвеев В. Б., Новиков С. П. Нелинейные уравнения типа Кортевега-де Фриза, конечнозонные линейные операторы и абелевы многообразия // УМН. 1976. T. 31. № 1. C. 55-136.

[9] Веселов А. П. Интегрируемые системы с дискретным временем и разностные операторы // Функцион. анализ и его прилож. 1988. Т. 22. № 2. С. 1-13.

[10] Magri F. A simple model of integrable Hamiltonian equation // J. Math. Phys. 1978. V. 19. P. 1156-1162. 